\title{
Thematic Tactile Cartography: Evaluating Tactile Mapping Techniques for Novel Applications
}

\author{
Harrison Cole ${ }^{a}$ \\ ${ }^{a}$ Department of Geography, The Pennsylvania State University, harrisoncole@psu.edu
}

Keywords: Tactile cartography, thematic cartography, usability, accessibility, flood maps

\begin{abstract}
:
Research on tactile maps for blind people has historically been a largely utilitarian endeavour, with research questions focusing on either legibility of the maps themselves or applying the map's data to clear, practical goals, such as wayfinding (Cole, 2021). Tasks that require higher-order analytical thinking - such as exploratory data analysis, decisionmaking, or even simple comparative spatial analysis - are not entirely absent in tactile cartography research, but are notably less common. There are three important factors that help explain this tendency. First, tactile maps are often more difficult to create than visual maps, both in terms of design and actual production (Brittell, Lobben, \& Lawrence, 2018). In most cases, the amount of information that one is able to present on a tactile map is significantly less extensive than visual maps. Thus, selection, generalization, and simplification are all much more consequential processes and often take longer. Second, thematic cartography - and to a larger degree GIS analysis - relies on the "layering" of data in order to reveal spatial patterns. While this is easily represented with conventional visual graphics, the tools that one might use to suggest superimposition, such as transparency, animation, or perspective, might end up obfuscating data in a tactile map, or are simply not possible to apply. Third, despite several decades of calls for the standardization of tactile mapping practices, cartographers have yet to compile a comprehensive set of best practices, symbols, or methods (Rahardjo, M, \& Kartika, 2019). Instead, guidelines that have been developed for tactile graphics more generally are simply applied to maps and adjusted when necessary. While many of the same principles of accessible graphics do indeed apply to maps which, after all, are a graphical document, their impact on spatial cognition is less certain. That is to say, it is an open question whether or not - or to what degree - tactile maps should have their own design conventions independent of other types of tactile graphics.
\end{abstract}

I present my research as a starting point for addressing these issues. Rather than propose another novel technology or design for tactile cartography, I instead evaluate existing cartographic methods in a novel context- namely, as they apply to higher-order analytical tasks. Because of the subjective nature of these tasks, evaluation will not exclusively determine whether or not the participants correctly interpret data, but rather will evaluate the confidence of blind users in their ability to use tactile maps for analysing and synthesizing spatial information. The scenario that I use for these evaluations is that of flood risk mitigation planning.

In the United States, the Federal Emergency Management Agency (FEMA) distributes funds to local government agencies if they submit a hazard mitigation plan (HMP), which describes the steps that the community will take to address various natural and manmade hazards. In the case of floods in particular, HMPs make extensive use of maps, especially because insurance rates are greatly affected by a building's proximity to flood zones. Thus, special maps called flood insurance rate maps (FIRMs) are used in the hazard mitigation planning process in order to illustrate the extent and character of flood risk in a city. An important component of the HMP process is the solicitation of input from community stakeholders and the general public. In any given city, these community members may be blind or visually impaired, which of course means that visual FIRMs would be of limited to no use for them, thus limiting their capacity to meaningfully participate in the HMP process. Given the millions of people who live with some sort of visual impairment in the US, the input of people from this demographic is of no small importance for ensuring the safety and integrity of communities.

My studies entail the creation of tactile FIRMs using a combination of tools and design conventions that have been developed for tactile wayfinding maps and non-cartographic tactile graphics. Participants (who are blind) are given two maps. The first map is based on parameters used by Lighthouse San Francisco TMAP (Tactile Map Automated Production) software, which creates tactile wayfinding maps (Miele, Landau, \& Gilden, 2006). Building and hydrographic data is added to the map. The second map (Figure 1) will be the same as the first, but with the addition of flood zone polygons based on FIRM data. Symbols for flood zones are based on consultation with professional tactile graphics designers, users of tactile graphics, and guidance outlined by the Braille Authority of North America (BANA). While the maps are based on real-world locations, they are not meant to be locations familiar to participants, in order to ensure that the maps are being evaluated rather than participant knowledge. Participants then complete tasks that emulate the type of analysis that would be required during real-world hazard mitigation planning. Example tasks include characterizing flood zones, assessing the potential need for more accessible infrastructure, and comparing vulnerability between multiple areas represented in the maps to direct flows of resources. 


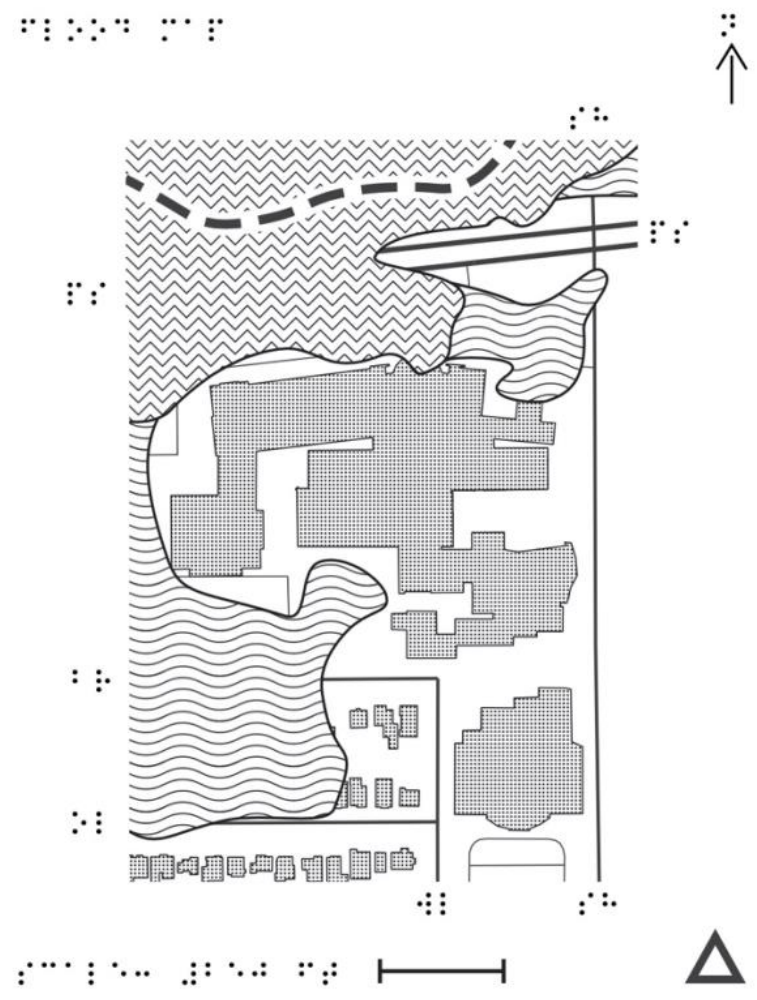

Figure 1. Design of a prototype tactile flood map. Solid lines represent roads, polygons with gridded dots represent buildings, polygons with repeating lines represent flood zones, and the large, dashed line represents a river.

After using the maps, participants respond to a survey that asks them to rank how confident they were in performing the requested tasks. High confidence suggests that existing techniques for creating tactile maps may in fact be sufficient for analytical spatial thinking, and future accessibility research needs to focus not on technical development, but dissemination and adaptation, or in other words, getting these maps into the hands of the people who need them. On the other hand, low confidence while using tactile FIRMs suggests that design and media need to be developed further in order to meet the needs of analytical spatial thinking.

In my talk, I present the results of my evaluative studies and give recommendations for future directions that research may take. Many of the most pressing issues that humans face - climate change, pandemics, natural resource depletion and so on - are not only intrinsically spatial, but multiscalar. Thus, it is critical to continue developing and distributing tools for helping people understand the spatial nuances of these issues as they pertain to their own communities and the communities of others. If a significant portion of the world's population is unable to use these tools, then we lose out, as a whole, on unique insight, knowledge, and experiences that could improve and even save lives.

\section{References:}

Brittell, M. E., Lobben, A. K., \& Lawrence, M. M. (2018). Usability Evaluation of Tactile Map Symbols across Three Production Technologies. Journal of Visual Impairment \& Blindness, 112(6), 745-758.

https://doi.org/10.1177/0145482X1811200609

Cole, H. (2021). Tactile cartography in the digital age: A review and research agenda: Progress in Human Geography. (Sage UK: London, England). https://doi.org/10.1177/0309132521995877

Miele, J. A., Landau, S., \& Gilden, D. (2006). Talking TMAP: Automated generation of audio-tactile maps using Smith-Kettlewell's TMAP software. British Journal of Visual Impairment, 24(2), 93-100. https://doi.org/10.1177/0264619606064436

Rahardjo, N., M, I. N. M., \& Kartika, C. S. D. (2019). Specifications of Cartographic Symbols for Indonesian Tactile Map. Indonesian Journal of Geography, 51(1), 62. https://doi.org/10.22146/ijg.41960 